\title{
Promoter and transcription factor dynamics tune protein mean and noise strength in a quorum sensing-based feedback synthetic circuit
}

\author{
Yadira Boada $^{1, \infty a}$, Alejandro Vignoni ${ }^{2, ~ a b ~}{ }^{\text {Jesús Picó }}{ }^{1, \infty a}{ }^{*}$, \\ 1 Institut d'Automàtica i Informàtica Industrial, Universitat Politècnica de \\ València, Spain \\ 2 Center for Systems Biology Dresden, Max Planck Institute of Molecular \\ Cell Biology and Genetics, Dresden, Germany
}

Ca Camino de Vera s/n, 46022, Valencia, Spain.

, $\mathrm{b}$ Pfotenhaurstr. 108, 01307 Dresden, Germany.

* E-mail: jpico@upv.es

\begin{abstract}
Gene expression is a fundamental cellular process. Its stochastic fluctuations due to intrinsic and extrinsic sources, known generically as 'gene expression noise', trigger both beneficial and harmful consequences for the cell behavior.

Controlling gene expression noise is of interest in many applications in biotechnology, biomedicine and others. Yet, control of the mean expression level is an equally desirable goal. Here, we analyze a gene synthetic network designed to reduce gene expression noise while achieving a desired mean expression level. The circuit combines a negative feedback loop over the gene of interest, and a cell-to-cell communication mechanism based on quorum sensing. We analyze the ability of the circuit to reduce noise as a function of parameters that can be tuned in the wet-lab, and the role quorum sensing plays. Intrinsic noise is generated by the inherent stochasticity of biochemical reactions. On the other hand, extrinsic noise is due to variability in the cell environment and the amounts of cellular components that affect gene expression. We develop a realistic model of the gene synthetic circuit over the population of cells using mass action kinetics and the stochastic Chemical Langevin Equation to include intrinsic noise, with parameters drawn from a distribution to account for extrinsic noise. Stochastic simulations allow us to quantify the mean expression level and noise strength of all species under different scenarios, showing good agreement with system-wide available experimental data of protein abundance and noise in E. coli. Our in silico experiments reveal significant noise attenuation in gene expression through the interplay between quorum sensing and the negative feedback, allowing control of the mean expression and variance of the protein of interest. These in silico conclusions are validated by preliminary experimental results. This gene network could have important implications as a robust protein production system in industrial biotechnology.
\end{abstract}

\section{Author Summary}

Controlling gene expression level is of interest in many applications in biotechnology, biomedicine and others. Yet, the stochastic nature of biochemical reactions plays an 
important role in biological systems, and cannot be disregarded. Gene expression noise resulting from this stochasticity has been studied over the past years both in vivo, and in silico using mathematical models. Nowadays, synthetic biology approaches allow to design novel biological circuits, drawing on principles elucidated from biology and engineering, for the purpose of decoupled control of mean gene expression and its variance. We propose a gene synthetic circuit with these characteristics, using negative feedback and quorum sensing based cell-to-cell communication to induce population consensus. Our in silico analysis using stochastic simulations with a realistic model reveal significant noise attenuation in gene expression through the interplay between quorum sensing and the negative feedback, allowing control of the mean expression and variance of the protein of interest. Preliminary in vivo results fully agree with the computational ones.

\section{Introduction}

Noise due to stochastic phenomena is pervasive in the cellular mechanisms underlying gene expression 1, 2. Its consequences can trigger both detrimental and advantageous effects, and may determine the fate of individual cells and that of a whole population of cells $[3-6]$. The fluctuations in gene expression of single cells propagate to generate fluctuations in downstream genes affecting stress response, metabolism, development, cell cycle, etc. 5 7], and eventually are the cause of phenotypic noise, that is, variation within an isogenic population.

Experimental measurements of individual genes show that protein production occurs in bursts 4,8 10] that can be traced back to two main sources: intrinsic, and extrinsic noise. Intrinsic noise is due to stochastic fluctuations in the transcription and translation steps of a particular gene [11]. Thus, intrinsic noise in a gene is correlated with the characteristics of the gene promoter, ribosome binding site (RBS), and the stability of the mRNA and the expressed protein 12, 13. Transcription dominates the intrinsic noise as the burst size, i.e. the average number of proteins made per mRNA transcript, increases [14]. On the other hand, extrinsic noise corresponds to gene independent fluctuations in protein expression due to external factors like gene copy numbers, transcription factor and ribosome abundance, and/or environmental stimuli [11]. When the gene is encoded on a low-copy plasmid, variability in the gene copy number is a major source of extrinsic noise [15. This may dominate in eukaryotes [16, while in prokaryotes seems only contribute increasing the noise floor 17 .

To minimize the deleterious effects of noise, cells use specific biochemical networks. At the most basic level, cells have evolved different transcription and translation efficiency so as to reduce translation burst rates in key genes 2, 9, 18. More elaborated strategies, such as negative feedback regulation, may reduce noise by shifting the noise spectrum to a higher frequency region [1,19. Ultrasensitive switches and feedforward loops are able to attenuate noise in input signals [20]. These strategies operate at the single-cell level. Yet, cells live in communities, forming a population. At this level, extracellular signaling propagates intracellular stochastic fluctuations across the population 21. Thus, bacteria have adapted their communication mechanisms in order to improve the signal-to-noise ratio [22]. One of such communication mechanisms is quorum sensing (QS).

Quorum sensing is a cell-to-cell communication mechanism initially discovered in $V$. fisheri and P. putida 23 24]. Bacteria release chemical signaling molecules, called autoinducers, whose external concentration increases as a function of the cell population density. Cells detect a threshold concentration of these autoinducers and alter gene expression accordingly 25 27. This strategy makes the population as a whole to achieve a desired gene expression level despite the individual noise of each member of 
the population. It is known that synchronization and consensus protect from noise 28 . Cells consensus induced by diffusion of the signaling autoinducer reduces extrinsic noise by reducing the transmission of fluctuating signals (including noise) in the

low-frequency domain 20, and enhances intrinsic stochastic fluctuations 21. Moreover, quorum sensing allows entrainment of a noisy population when faced to environmental changing signals 29 . Therefore QS seems an effective tool to control the phenotypic variability in a population of cells [30].

Phenotypic variability has important practical relevance in many applications in the areas of biomedicine, biotechnology and other branches of biological science [31. In particular, the presence of heterogeneous subpopulations may have significant impact on the yield and productivity of industrial cultures 3234 . Thus, improving homogeneity of protein expression in industrial cultures is a goal of economic relevance for microbial cell factory processes.

Improving homogeneity of protein expression has traditionally been attempted either by optimizing environmental conditions in the culture or by careful selection of the strain. Yet, there is an ever-growing appreciation that biological complexity requires new bioprocess design principles. Synthetic biology, sometimes defined as the engineering of biology, has the potential to engineer genetic circuits to perform new functions for useful purposes in a systematic, predictable, robust, and efficient way [35, 36]. In the last years, several synthetic circuits have been proposed with the ultimate goal of dealing with gene expression noise $20,22,37,40$.

Synthetic biology makes extensive use of mathematical models and computational simulation to aid the genetic circuit design. They allow the generation of new testable hypotheses and novel ways of intervention, and offer mechanistic explanations of experimental results. The dynamics of the reactions involved in gene expression have been traditionally described using continuous deterministic mathematical models 41,42 . These equations can be easily obtained from the reactions using the law of mass action kinetics [43. However, this continuous deterministic approach fails to capture the consequences of stochasticity in gene expression. Hence, there is an extensive literature on rigorous modeling of gene expression noise and determination of its sources [44].

The most accurate way to represent the stochasticity originated from intrinsic sources is by means of the Chemical Master Equation (CME). The CME determines the probability that each species will have a specified number of molecules at each time instant 44 45]. Though the CME can be solved analytically for small systems [46 47, it is in general not a tractable problem, or even possible, for systems of medium to large size. Not even numerically. Several alternatives have been derived with a broad range of computational cost and precision. Some numerical schemes like those in 48, 49, provide a solution of the CME in a truncated state-space. However, long-term predictions are not always possible, specially for bimolecular reactions. In 50 the authors present a modified CME based on the partition of a system into reactions with low and high propensity, thus providing approximations at different levels of accuracy and computational cost. The Gillespie SSA algorithm [51] is a widely used Monte Carlo-based approach that provides exact samples from the probability distribution that results from solving the CME. However, having an interconnected population of cells, as in our proposed gene synthetic circuit, jeopardizes the possibility of employing SSA for several reasons. First there are different volumes involved, extracellular and intracellular. The diffusion through the membrane of the autoinducer molecule used for cell-to-cell communication depends on its concentration in both of them, making the account for the probability of reaction more complicated. Second, when using SSA, several realizations or trajectories of the system are needed in order to obtain an accurate estimation of the statistical moments of the species in the circuit, making the use of SSA in a population of interconnected cells a computationally very demanding task. At 
the opposite extreme the linear noise approximation (LNA) allows to get the decoupled dynamics of the mean and variance of gene expression [19,52,53] using a first order approximation (i.e. a linearization) of the difference between the actual noisy trajectory, and the mean deterministic one. An intermediate practical alternative to model gene expression intrinsic noise is the use of the Chemical Langevin Equation (CLE).

The CLE is a stochastic differential equation (SDE) driven by zero-mean Gaussian noise that describes the system when the molecules of reactants into a cell population are sufficiently large 54,55 . It approximates the CME by a system of stochastic differential equations of order equal to the number of species $-c f$. order equal to the possible number of molecules of all the species in the CME. Extrinsic noise can be modeled by randomizing values of the model parameters [16 56], an approach that can easily be integrated within the CLE framework. The CLE has also been used to study intrinsic noise in synthetic systems involving QS mechanisms in 20 though the authors considered an averaged cell, thus not taking into account single cell contributions to the noise in the population.

In this work we analyze a gene synthetic network designed to reduce gene expression noise while achieving a desired mean expression level. The circuit combines a negative feedback loop over the gene of interest, and a cell-to-cell communication mechanism based on quorum sensing. In the Materials and Methods Section, we develop a realistic model of the gene synthetic network over the population of cells using mass action kinetics. Then, stochastic simulations using the Chemical Langevin Equation allow us to quantify the noise strength of all species under different scenarios. In particular, in the Results Section we analyze the ability of the circuit to reduce noise as a function of parameters that can be tuned in the wet-lab, and the role quorum sensing plays. We show preliminary experimental data validating our in silico results, and finally we draw some conclusions in the Discussion Section.

\section{Materials and Methods}

\section{Description of the synthetic gene network.}

Reducing gene expression noise at the level of an individual cell can be attempted in several ways. Open loop strategies as based on sensitivity analysis providing guides as to how properly tune transcriptional and translational parameters so that the noise levels can be controlled while the mean values can be simultaneously adjusted to desired values [57. While sensitivity analysis gives very valuable insights, open loop control is not robust against system uncertainty and/or variations. On the other hand, closed loop control can be implemented by using a negative feedback loop over the gene of interest and appropriately tuning it. Though negative feedback has been proved to decrease gene expression noise 37, a single-cell intracellular feedback loops do not take into account that in practice one is interested in controlling gene expression mean value and noise across a population of cells. Feedback across a population of cells can be implemented by means of quorum sensing-based strategies, and has been shown to reduce noise effects 20,22 30]. Indeed, cell-to-cell communication by means of quorum sensing induces consensus among cells [58], that is contributes to reduce the difference of internal state among cells in a population. This, in turn, contributes to protect from noise 28. Thus, the idea of joining both intracellular negative feedback and extracellular feedback via quorum sensing is a natural one, that has been suggested in 38,39 .

Here we analyze the gene synthetic network proposed in [38], designed to reduce gene expression noise while achieving a desired mean expression level. The circuit combines a negative feedback loop over the gene of interest, and a cell-to-cell 
communication mechanism based on quorum sensing. For that purpose, the circuit employs two functional subsystems already implemented in E. coli (see Fig 11A).

A

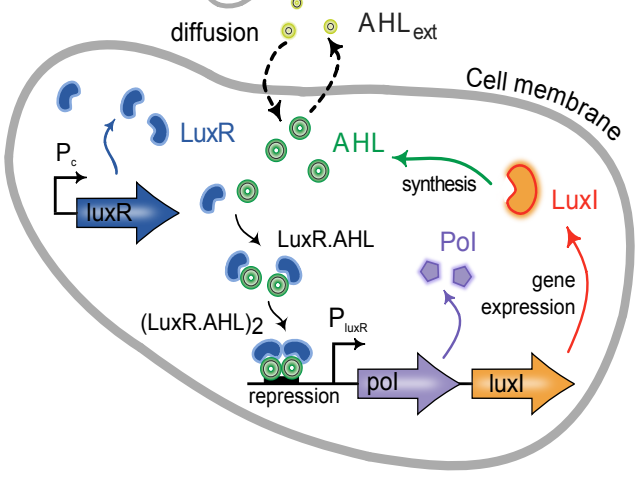

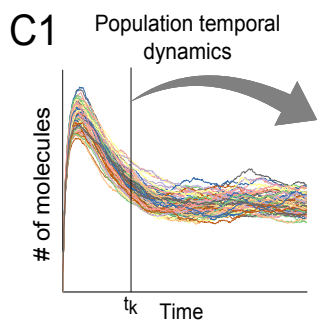

C4 Noise strength map

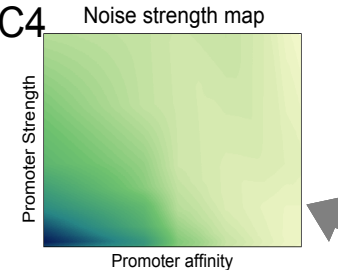

C3 Long-term distribution

C2 Population distribution
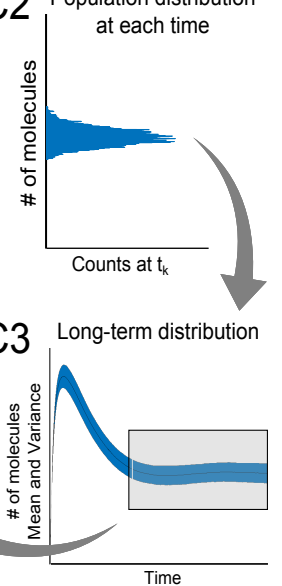

B

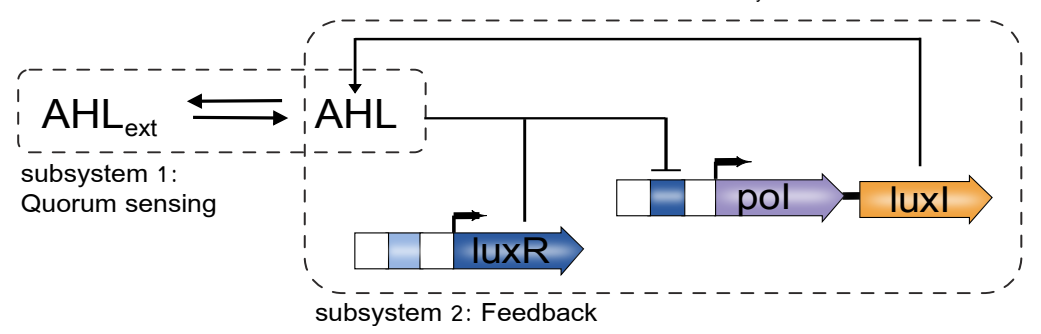

Figure 1. Synthetic gene network. A. Intracellular and extracellular system with negative feedback control and quorum sensing mechanism. B. Biochemical reactions and diffusion process. C. Methodological procedure to obtain the statistical moments from stochastic simulations of the circuit. C1) Temporal evolution of one species in the population of cells. C2) Distribution of the number of molecules across the population at each time instant. C3) Acquisition of the long-term distribution for each species. C4) Noise strength map for varying model parameters.

The first subsystem implements a cell-to-cell communication mechanism via quorum sensing, based on exchange of the small signaling autoinducer molecule N-acyl-L-homoserine lactone (AHL) [25|26]. This autoinducer molecule passively diffuses across the cellular membrane to and from the external environment. Intracellular AHL is synthesized by the protein LuxI expressed by an homolog of the gene luxI of $V$. fisheri [59].

The second subsystem uses the synthetic repressible promoter $\mathrm{P}_{\text {luxR }}$ designed in 60 to control transcription of gene luxI. This promoter is repressed by the transcription factor (LuxR.AHL) ${ }_{2}$. Protein LuxR is expressed by gene luxR under the constitutive promoter $\mathrm{P}_{\mathrm{c}}$. Proteins LuxR and AHL bind forming the heterodimer (LuxR.AHL), which subsequently dimerizes forming the heterotetramer (LuxR.AHL) $)_{2}$. This way a negative feedback control of the LuxI expression is effectively implemented 38 .

The circuit acts as a closed loop controller of the mean and variance of a protein of interest. This protein can be either fused to protein LuxI, or coexpressed with it. In the first case, a linker is inserted between the fused proteins allowing intracellular self-cleavage using a TEV protease $61-63$. Alternatively, if the protein of interest is coexpressed with LuxI, the controller will only act at the transcriptional level. In cases where transcriptional noise dominates translational one, e.g. when the average number 
of proteins made per mRNA transcript is larger than two [14, coexpressing LuxI with the protein of interest is a simple yet effective approach.

Note: Henceforth, and for the sake of simplicity, we will call monomer the heterodimer (LuxR · AHL), and dimer the heterotetramer transcription factor $(\mathrm{LuxR} \cdot \mathrm{AHL})_{2}$.

\section{Mathematical model.}

In order to analyze how our genetic circuit affects intrinsic and extrinsic noise we need an appropriate model, and a computationally efficient method. Both aspects are intertwined. We use the Chemical Langevin Equation approach. Though computationally much more efficient than the CME or even the Gillespie algorithm, the CLE is still computationally demanding when the goal is to simulate a whole population of cells. Since the CLE approximates the CME by a system of stochastic differential equations of order equal to the number of species, a reduced model with as few species per cell as possible is desirable. Thus, in a first step we use the mass-action kinetics formalism [42,43] to get a deterministic model of the full reactions network corresponding to the genetic circuit. We then get a reduced order model by applying the Quasi Steady-State Approximation (QSSA) on the fast chemical reactions and taking into account invariant moieties $53,64,65$. We aim at obtaining a reduced model more amenable for computational analysis, but avoiding excessive reduction that would lead to lack of biological relevance. In particular, the species we obtain in the reduced are not lumped ones. Reduced models accounting for total mRNA and total transcription factor have been proposed to match modeled species with measurable ones [66]. In our case we explicitly model bound and unbound forms of the transcription factor, but the model accounts for the total LuxI protein. For our circuit this is a good proxy of the amount of protein of interest if both are co-expressed, and transcriptional noise dominates. In the best case, when the protein of interest is in self-cleavable tandem fusion with LuxI, both will express in 1:1 stoichiometric ratio 62. Moreover, the resulting lumped parameters in the reduced model are easy to associate to tuning knobs available in the wet-lab implementation in the relevant cases [67], and their values are amenable to be obtained experimentally.

In a second step we use the deterministic reduced model to infer a stochastic CLE-based model whose mean corresponds to that of the deterministic one.

Reduced deterministic model. We start from considering the relevant biochemical reactions in the circuit. We consider the gene expression sets of reactions on the one hand, and the induction ones on the other. In the gene expression block, the main processes considered for each of the proteins are transcription, translation, mRNA degradation and protein degradation. In the induction block, the reactions considered are reversible binding between the protein LuxR and the inducer AHL to form the monomer (LuxR.AHL), monomer degradation, reversible dimer (LuxR.AHL) $)_{2}$ formation and its degradation, diffusion of the inducer across the cell membrane, extracellular and intracellular inducer degradation, and reversible binding of the dimer to the repressible promoter $\mathrm{P}_{\text {luxR }}$. We assumed the promoter $\mathrm{P}_{\text {luxR }}$ is leaky.

From the resulting set of reactions, and based on mass-action kinetics we derived a complete deterministic model using ordinary differential equations (ODE) for number of molecules of each species. We considered the net effective transcription rates of genes luxI and luxR, and took into account the basal transcription rate (leakage) of the repressible promoter $\mathrm{P}_{\text {luxR }}$. Besides, the dilution effect due to the cells growth rate was added to every degradation rate. This complete model was then reduced using QSSA, conservation of moieties, and assuming that translation, diffusion across the cell 
Table 1. Parameters of the gene synthetic circuit model.

\begin{tabular}{|c|c|c|c|c|}
\hline Parameter & Description & Value & Unit & Reference \\
\hline $\mathrm{C}_{\mathrm{R}}$ & Plasmid copy number times LuxR transcription rate & $7.9^{\dagger}$ & molecules/min & 68 \\
\hline $\mathrm{C}_{\mathrm{I}}$ & Plasmid copy number times LuxI transcription rate & $17.5^{\dagger}$ & molecules/min & 68 \\
\hline$\alpha$ & Basal expression of luxI & 0.01 & & estimated \\
\hline $\mathrm{p}_{\mathrm{R}}$ & Translation rate of $\mathrm{mRNA}_{\mathrm{LuxR}}$ & $2.38^{\ddagger}$ & $\min ^{-1}$ & {$[\overline{42,}, \overline{69}$} \\
\hline $\mathrm{p}_{\mathrm{I}}$ & Translation rate of $\mathrm{mRNA}_{\mathrm{LuxI}}$ & $3.09^{\ddagger}$ & $\min ^{-1}$ & $\overline{42,}, \overline{69}$ \\
\hline $\mathrm{k}_{\mathrm{A}}$ & Synthesis rate of AHL by LuxI & 0.04 & $\min ^{-1}$ & 38 \\
\hline $\mathrm{k}_{-1}$ & Dissociation rate of $(\mathrm{LuxR} \cdot \mathrm{AHL})$ & 10 & $\min ^{-1}$ & 30] \\
\hline $\mathrm{k}_{-2}$ & Dissociation rate of dimer $(\mathrm{LuxR} \cdot \mathrm{AHL})_{2}$ & 1 & $\min ^{-1}$ & estimated \\
\hline $\mathrm{k}_{\mathrm{d} 1}$ & Dissociation constant of (LuxR $\cdot$ AHL) & 100 & molecules & 70 \\
\hline $\mathrm{k}_{\mathrm{d} 2}$ & Dissociation constant of $(\mathrm{LuxR} \cdot \mathrm{AHL})_{2}$ & 20 & molecules & $71]$ \\
\hline $\mathrm{k}_{\mathrm{dlux}}$ & Dissociation constant of (LuxR $\cdot \mathrm{AHL})_{2}$ to the lux promoter & 100 & molecules & 72] and refs. therein \\
\hline $\mathrm{d}_{\mathrm{I}}$ & Degradation rate of LuxI & $0.027^{b}$ & $\min ^{-1}$ & 69,73 \\
\hline $\mathrm{d}_{\mathrm{R}}$ & Degradation rate of LuxR & $0.156^{b}$ & $\min ^{-1}$ & 74] and refs. therein \\
\hline $\mathrm{d}_{\mathrm{A}}$ & Degradation rate of AHL & $0.057^{b}$ & $\min ^{-1}$ & {$[\overline{59}, \overline{75}$} \\
\hline $\mathrm{d}_{\mathrm{A}_{\mathrm{e}}}$ & Degradation rate AHL in culture medium & 0.04 & $\min ^{-1}$ & $25,59,75]$ \\
\hline $\mathrm{d}_{\mathrm{RA}}$ & Degradation rate of $($ LuxR $\cdot$ AHL $)$ & $0.156^{b}$ & $\min ^{-1}$ & 72] and refs. therein \\
\hline $\mathrm{d}_{\mathrm{RA}_{2}}$ & Degradation rate of $(\mathrm{LuxR} \cdot \mathrm{AHL})_{2}$ & 0.017 & $\min ^{-1}$ & estimated \\
\hline $\mathrm{dm}_{\mathrm{I}}$ & Degradation rate of $\mathrm{mRNA}_{\text {LuxI }}$ & $0.247^{b}$ & $\min ^{-1}$ & 76.77 \\
\hline $\mathrm{dm}_{\mathrm{R}}$ & Degradation rate of $\mathrm{mRNA}_{\mathrm{LuxR}}$ & $0.247^{b}$ & $\min ^{-1}$ & 69.77 \\
\hline $\mathrm{D}$ & Diffusion rate of AHL through the cell membrane & $2^{4}$ & $\min ^{-1}$ & $\overline{78}, \overline{79}$ \\
\hline $\mathrm{V}_{\text {cell }}$ & Typical volume of E. coli. & $1.1 e-9$ & $\mu \mathrm{L} /$ cell & $69]$ \\
\hline $\mathrm{V}_{\text {ext }}$ & Typical volume of microfluidic device & $1 e-3$ & $\mu \mathrm{L}$ & estimated \\
\hline
\end{tabular}

${ }^{\dagger}$ The parameters $C_{R}$ and $C_{I}$ can be tuned by selecting the strength of the promoter and/or using plasmids with different copy number. We used the typical transcription rate in E. coli. $\approx 10-100 \mathrm{bp} / \mathrm{sec} 80]$, and considered the number of base pairs of the coding sequences for genes luxI and luxR. The resulting rate was multiplied by the plasmid copy number. We assumed a low plasmid copy number in the interval 10-20 69,81].

$\ddagger$ The translation rate can be tuned using ribosome-binding site (RBS) of different strengths. In bacteria, the translation rate is $\approx$ $30-60 \mathrm{bp} / \mathrm{sec}$ and the average number of ribosomes per cell (in the exponential phase) to translate one mRNA is $\approx 9$ units.

b These degradation rates include the dilution effect due to the specific growth rate $\mu_{\text {spe }}=0.017 \mathrm{~min}^{-1}$ corresponding to a cell

doubling time $\approx 40 \mathrm{~min}$. The degradation rate $\mathrm{d}_{\mathrm{RA}_{2}}=\mu_{\text {spe }}$ assuming the dimer is much more stable than the other species 72,82$]$.

I The diffusion coefficient $\mathrm{D}=\frac{\mathrm{SP}_{\mathrm{n}}}{\mathrm{V}_{\text {cell }}} \mathrm{min}^{-1}$ depends on the cell surface area $\mathrm{S}=4 \pi \mathrm{r}^{2}$ (spherical area with $\mathrm{r}=10 \mu \mathrm{m}$ ), the membrane permeability $\mathrm{P}_{\mathrm{n}}=3 \mathrm{e}^{-3} \mu \mathrm{m} / \mathrm{min}$ and the cell volume $\mathrm{V}_{\text {cell }}$.

membrane, and dimerization are the dominant dynamics. With this assumptions we got the dynamic ODE model:

$$
\begin{aligned}
& \dot{n}_{1}^{i}=\frac{\mathrm{C}_{\mathrm{I}} \mathrm{p}_{\mathrm{I}}}{\mathrm{dm}_{\mathrm{I}}}\left(\frac{\mathrm{k}_{\mathrm{dlux}}+\alpha n_{3}^{i}}{\mathrm{k}_{\mathrm{dlux}}+n_{3}^{i}}\right)-\mathrm{d}_{\mathrm{I}} n_{1}^{i} \\
& \dot{n}_{2}^{i}=\frac{\mathrm{C}_{\mathrm{R}} \mathrm{p}_{\mathrm{R}}}{\mathrm{dm}_{\mathrm{R}}}+\mathrm{k}_{-1} n_{6}^{i}-\left(\frac{\mathrm{k}_{-1}}{\mathrm{k}_{\mathrm{d} 1}} n_{4}^{i}+\mathrm{d}_{\mathrm{R}}\right) n_{2}^{i} \\
& \dot{n}_{3}^{i}=\frac{\mathrm{k}_{-2}}{\mathrm{k}_{\mathrm{d} 2}}\left(n_{6}^{i}\right)^{2}-\left(\mathrm{k}_{-2}+\mathrm{d}_{\mathrm{RA}_{2}}\right) n_{3}^{i} \\
& \dot{n}_{4}^{i}=\mathrm{k}_{-1} n_{6}^{i}+\mathrm{k}_{\mathrm{A}} n_{1}^{i}+\mathrm{D}\left(\frac{\mathrm{V}_{\text {cell }}}{\mathrm{V}_{\text {ext }}} n_{5}-n_{4}^{i}\right)-\left(\frac{\mathrm{k}_{-1}}{\mathrm{k}_{\mathrm{d} 1}} n_{2}^{i}+\mathrm{d}_{\mathrm{A}}\right) n_{4}^{i} \\
& \dot{n}_{5}=\mathrm{D}\left(-N \frac{\mathrm{V}_{\text {cell }}}{\mathrm{V}_{\text {ext }}} n_{5}+\sum_{i=1}^{N} n_{4}^{i}\right)-\mathrm{d}_{\mathrm{A}_{\mathrm{e}}} n_{5}
\end{aligned}
$$

with:

$$
n_{6}^{i}=\frac{\mathrm{k}_{\mathrm{d} 2}\left(\mathrm{~d}_{\mathrm{RA}}+\mathrm{k}_{-1}\right)}{4 \mathrm{k}_{2}}\left[\sqrt{\frac{8 \mathrm{k}_{-2}\left(2 \mathrm{k}_{-2} \mathrm{k}_{\mathrm{d} 1} n_{3}^{i}+\mathrm{k}_{-1} n_{2}^{i} n_{4}^{i}\right)}{\mathrm{k}_{\mathrm{d} 1} \mathrm{k}_{\mathrm{d} 2}\left(\mathrm{~d}_{\mathrm{RA}}+\mathrm{k}_{-1}\right)^{2}}+1}-1\right]
$$

where $n_{1}^{i}$ is the number of molecules of protein LuxI in the i-th cell, $n_{2}^{i}$ that of LuxR, $n_{3}^{i}$ is the dimer repressor (LuxR.AHL) $)_{2}, n_{4}^{i}$ is the intracellular amount of inducer AHL, $n_{5}$ is the external one, and $n_{6}^{i}$ is the amount of monomer (LuxR.AHL) molecules. Notice this is given as an algebraic equation obtained assuming that monomerization is faster than dimerization, and can be assumed to be at quasi-steady state, as confirmed by the comparison between the complete and the reduced model dynamics. Table 1 describes the parameters in the model and its nominal values. 
From the deterministic ODE model to the stochastic CLE one. To model gene expression intrinsic noise we derive a stochastic CLE-based model whose mean corresponds to that of the deterministic model (1)-(2). This can be done by considering an equivalent set of pseudo-reactions for the deterministic model. From these, one can set model (3), corresponding to the Euler-Maruyama discretization of the CLE for a system with a population of $N$ cells:

$$
\mathbf{n}(t+\delta t)=\mathbf{n}(t)+\mathbf{S} \cdot \mathbf{a}(\mathbf{n}) \delta t+\mathbf{S} \cdot \mathcal{N} \cdot \sqrt{\mathbf{a}(\mathbf{n})} \sqrt{\delta t},
$$

where $\mathbf{n}(t)=\left[\mathbf{n}(t)^{i}, \ldots n(t)^{\mathrm{N}}, n_{5}\right]^{T}$ are the number of molecules of each species in the population, being $\mathbf{n}(t)^{i}$ the vector of species LuxI, LuxR, (LuxR.AHL) $)_{2}$, and intracellular AHL for the $i^{\text {th }}$ cell, and $n_{5}$ the extracellular one $\mathrm{AHL}_{\text {ext }}$. The stoichiometry matrix $\mathbf{S}$, whose elements are the stoichiometric matrices of each cell $\mathbf{S}_{\text {cell }}$ and the external stoichiometry $\mathbf{S}_{\mathbf{e x t}}$, has structure:

$$
\mathbf{S}=\left[\begin{array}{c|c}
\mathbf{S}_{\mathbf{c e l l}} \otimes \mathbf{I}_{\mathbf{N}} & \mathbf{0}_{\mathbf{N} \times \mathbf{1}} \\
\hline \mathbf{S}_{\mathbf{e x t}} \otimes \mathbf{1}_{\mathbf{1} \times \mathbf{N}} & -1
\end{array}\right]
$$

where $\otimes$ is the Kronecker product, $\mathbf{I}_{\mathbf{N}}$ the identity matrix of dimension $N \times N, \mathbf{0}_{\mathbf{N} \times \mathbf{1}}$ and $\mathbf{1}_{\mathbf{1} \times \mathbf{N}}$ are vectors of zeroes and ones respectively, and matrices $\mathbf{S}_{\text {cell }}$ and $\mathbf{S}_{\mathbf{e x t}}$ are expressed by equations (5).

$$
\begin{aligned}
\mathbf{S}_{\mathbf{c e l l}} & =\left[\begin{array}{ccccccccccccc}
1 & -1 & 0 & 0 & 0 & 0 & 0 & 0 & 0 & 0 & 0 & 0 & 0 \\
0 & 0 & 1 & 1 & -1 & -1 & 0 & 0 & 0 & 0 & 0 & 0 & 0 \\
0 & 0 & 0 & 0 & 0 & 0 & 1 & -1 & -1 & 0 & 0 & 0 & 0 \\
0 & 0 & 0 & 1 & -1 & 0 & 0 & 0 & 0 & 1 & -1 & -1 & 1
\end{array}\right] \\
\mathbf{S}_{\text {ext }} & =\left[\begin{array}{lllllllllllll}
0 & 0 & 0 & 0 & 0 & 0 & 0 & 0 & 0 & 0 & 0 & 1 & -1
\end{array}\right] .
\end{aligned}
$$

The coefficients in the stoichiometric matrices (5) were obtained from the equivalent set of pseudo-reactions for the deterministic model, and the term $\mathbf{a}(\mathbf{n})$ in model $(3)$ is the associated vector of reaction propensities for the whole population of cells, with:

$$
\mathbf{a}(\mathbf{n})=\left[\begin{array}{c}
\mathbf{a}(\mathbf{n})^{1} \\
\mathbf{a}(\mathbf{n})^{2} \\
\vdots \\
\mathbf{a}(\mathbf{n})^{N} \\
\hline \mathrm{d}_{\mathrm{A}_{\mathrm{e}}} n_{5}
\end{array}\right]
$$

$$
\mathbf{a}(\mathbf{n})^{i}=\left[\begin{array}{c}
f\left(n_{3}^{i}, t\right) \\
\mathrm{d}_{\mathrm{I}} n_{1}^{i} \\
\frac{\mathrm{C}_{\mathrm{R}} \mathrm{pR}}{\mathrm{dm}_{\mathrm{R}}} \\
\mathrm{k}_{-1} n_{6}^{i} \\
\frac{\mathrm{k}_{-1}}{\mathrm{k}_{\mathrm{d} 1}} n_{2}^{i} n_{4}^{i} \\
\mathrm{~d}_{\mathrm{R}} n_{2}^{i} \\
\frac{\mathrm{k}_{-2}}{\mathrm{k}_{\mathrm{d} 2}}\left(n_{6}^{i}\right)^{2} \\
\mathrm{k}_{-2} n_{3}^{i} \\
\mathrm{~d}_{\mathrm{RA}} n_{3}^{i} \\
\mathrm{k}_{\mathrm{A}} n_{1}^{i} \\
\mathrm{~d}_{\mathrm{A}} n_{4}^{i} \\
\mathrm{D} n_{4}^{i} \\
\mathrm{DV} n_{5}
\end{array}\right]
$$

where $f\left(n_{3}\right) \triangleq \frac{\mathrm{C}_{\mathrm{I}} \mathrm{p}_{\mathrm{I}}}{\mathrm{dm}_{\mathrm{I}}}\left(\frac{\mathrm{k}_{\mathrm{dlux}}+\alpha n_{3}^{i}}{\mathrm{k}_{\mathrm{dlux}}+n_{3}^{i}}\right)$ is the Hill-like function associated to LuxI repression.

Finally, $\mathcal{N}_{(\mathbf{J N}+\mathbf{1}) \times(\mathbf{J N}+\mathbf{1})}$, where $J=13$ is the number of reactions for the $i^{\text {th }}$ cell, i.e. the dimension of $(7)$, is a diagonal matrix with continuous normal random variables of zero mean and unit variance as elements.

Notice we use lumped propensity functions in 7 derived from the reduced model, like the $f\left(n_{3}\right)$ Hill-like function associated to LuxI repression. This approach has already been used in 83 . We validated it for our model by simulating the pseudo-reaction associated to $f\left(n_{3}\right)$ using CLE, and comparing the result with that 
obtained by simulating the set of corresponding original reactions using Gillespie's direct method SSA.

Extrinsic noise can be modeled by randomizing the values of the model parameters 16, 56, an approach that can easily be integrated within the CLE framework. In particular, we assumed a normal distribution in the model parameters to account for the extrinsic noise.

\section{Computational analysis.}

We use the stochastic model (3) of the proposed circuit, hereafter denoted as circuit $\mathrm{Qs} / \mathrm{Fb}$, to explore the impact of some key circuit parameters on noise. As control circuit to compare with, we consider a second circuit which removes both QS and the feedback loop, denoted as NoQs/NoFb. For the computational analysis, this accounts to setting the synthesis of AHL to zero $\left(k_{\mathrm{A}}=0[1 / \mathrm{min}]\right)$ in model $(3)$. This condition is achieved in the lab experimental implementation by taking out the gene coding for LuxI (see ). To asses the effect of cell-to-cell communication, we also considered a hypothetical circuit with feedback but without quorum sensing (NoQS/Fb, $D=0$ ). Notice the circuit NoQS/Fb cannot actually be implemented for it assumes there is no diffusion of the autoinducer molecule across the cell membrane. Yet, it is useful as a computational thought experiment to account for the contribution of the cell-to-cell communication.

Gene expression noise is evaluated using the squared coefficient of variation, i.e. the noise strength measure $\left(\eta^{2}=(\sigma / \mu)^{2}\right)$. The noise strength measure $\eta^{2}$ properly captures the contributions of both intrinsic and extrinsic noise [84, and allows comparisons for different expression rates.

We followed the general procedure depicted in (Fig $1 \mathrm{C})$. First, for different combinations of the model parameters, we performed simulations of the temporal evolution of the number of molecules of each species in the circuit for each cell in the population involved in our system (Fig $1 \mathrm{C} 1$ ). Extrinsic noise was modeled by randomizing the values of the model parameters using a normal distribution with a variance of $15 \%$. The models were implemented using OpenFPM a $\mathrm{C}++$ version of the Parallel Particle Mesh (PPM) library allowing efficient computational particle-mesh simulations 85. In all simulations we used a population of $N=240$ cells in a culture volume of $10^{-3} \mu \mathrm{l}$, corresponding to an optical cell density $\mathrm{OD}_{600}=0.3$. Cell density variations did not appreciably change the results, confirming the results in [20].

Then, we obtained the first two statistical moments $\mu$ and $\sigma^{2}$ for each species in the cell population at every time $t_{\mathrm{k}}(\mathrm{Fig} 1 \mathrm{C} 2)$. We used the laws of total expectation and total variance. Using these moments, we calculated long-term distributions to infer the noise strength of each species (Fig $1 \mathrm{C} 3$ ). To this end, we checked with our models that one realization of the population of $N$ cells is enough to obtain unbiased values of the long-term moments of the population, provided there is enough time to perform the time average.

Finally, we generated noise strength maps for different sets of varying model parameters (Fig $1 \mathrm{C} 4$ ). We explored the effect of variations in parameters associated to expression of LuxI and LuxR, as they are as key parameters in our circuit. For LuxI we considered the dissociation constant $\mathrm{k}_{\mathrm{dlux}}$ between the transcription factor (LuxR - AHL $)_{2}$ and the repressible $\mathrm{P}_{\text {lux }}$ promoter, the translation rate $\mathrm{p}_{\mathrm{I}}$, and the basal expression $\alpha_{\mathrm{I}}$ of the $\mathrm{P}_{\text {lux }}$ promoter. We sampled in the ranges $\mathrm{k}_{\mathrm{dlux}}=[10-2000]$, $\alpha=[0.01-0.1]$, and $\mathrm{p}_{\mathrm{I}}=[0.2-10]$ selected from the literature 86.88 and experimentally achievable in the lab. As for LuxR, we considered two values for the the translation rate $\mathrm{p}_{\mathrm{R}}$ : a strong $\mathrm{RBS}\left(\mathrm{p}_{\mathrm{R}}=10\right)$, and a medium-weak one $\left(\mathrm{p}_{\mathrm{R}}=2\right)$. In

\footnotetext{
${ }^{1}$ Git repository available at https://github.com/incardon
} 
addition, we analyzed the effect of different degradation rates $d_{R}$ in the range $[0.02-0.2]$.

Notice from model (1) that although we only considered variations in the translation rates $\mathrm{p}_{\mathrm{I}}$ and $\mathrm{p}_{\mathrm{R}}$, these are tantamount to considering variations in the lumped values $\frac{C_{I} P_{I}}{d m_{I}}, \frac{C_{R} P_{R}}{d m_{R}}$ corresponding to the products of protein burst size, transcription rate and gene copy number. We assumed variations in translation rates just because they are relatively simple to modify in a graded way by tuning the RBS [86], though also transcription rates could be easily tuned [89].

\section{Plasmids and experimental conditions.}

To validate the in silico computational results, we implemented the $\mathrm{Qs} / \mathrm{Fb}$ and NQs/NFb circuits in vivo. We used components from the iGEM Registry of Standard Biological Parts (http://parts.igem.org). All parts were cloned using the Biobrick's foundation 3 Antibiotic Assembly method. All coding sequences have the double-terminator BBa_B0015, and were confirmed by sequencing.

The circuit Qs/Fb integrating both the QS-based cell-to-cell communication and the negative feedback subsystems, was split in two subunits integrated in different plasmids. On the one hand, plasmid pCB2tc contains the gene luxR (part BBa_C0062) coding for the protein LuxR constitutively expressed under the control of a medium strength promoter (part BBa_J23106) and a strong RBS (part BBa_B0034). This insert was cloned into the $\mathrm{pACYC} 184$ plasmid cloning vector (p15A origin, chloramphenicol/tetracycline). On the other hand, plasmid pYB06ta contains gene luxI (part BBa_C0161) under control of the $\mathrm{p}_{L u x R}$ repressible promoter (part BBa_R0062) and a strong RBS (part BBa_B0034). The strong RBS BBa_B0034 and the green fluorescent protein (GFP, part BBa_E0040) were inserted using GIBSON assembly (NEB Catalog \# E2611S) upstream of luxI, right after the $\mathrm{p}_{\text {LuxR }}$ promoter. This way, GFP, used as protein of interest (PoI in Fig 1 $)$ is co-expressed with LuxI. They were inserted into the pBR322 plasmid cloning vector (pMB1 origin, ampicillin/tetracycline). Finally, both plasmids pCB2tc and pYB06ta were co-transformed in competent cells (DH-5 $\alpha$, Invitrogen).

As control circuit, we implemented the circuit NQs/NFb which removes both QS and the feedback loop. To this end, the plasmid pCB2tc above was co-transformed with the plasmid pAV02ta (pMB1 origin, ampicillin/tetracycline) containing only GFP downstream the $\mathrm{p}_{\text {LuxR }}$ repressible promoter (part BBa_R0062) and the the strong RBS (part BBa_B0034). Both were cloned in the pBR322 plasmid cloning vector.

For the experimental validation of the circuit (see protocol details in Supplementary Text S3), two sets of E. coli cells (cloning strain DH-5 $\alpha$ ) carrying the Qs/Fb and $\mathrm{NoQs} / \mathrm{NoFb}$ circuits respectively were inoculated from $-80^{\circ} \mathrm{C}$ stocks into $3 \mathrm{~mL}$ of $\mathrm{LB}$ with appropriate antibiotics, followed by an overnight incubation at $37^{\circ} \mathrm{C}$ and $250 \mathrm{rpm}$ in $14 \mathrm{ml}$ culture tubes. When the cultures reached an optical density (OD) of 4 (600 $\mathrm{nm}$, Eppendorf BioPhotometer D30), the overnight cultures were diluted 500-fold (OD of 0.02 ) into M9 medium with appropriate antibiotics. These were used to inoculate new cultures, which were incubated for 7 hours $\left(37^{\circ} \mathrm{C}, 250 \mathrm{rpm}, 14 \mathrm{ml}\right.$ culture tubes) until they reached an OD between 0.2-0.3. At this point, cell growth and protein expression were interrupted by transferring the culture into an ice-water bath for 10 min. Next, $50 \mu \mathrm{L}$ of each tube were transferred into $1 \mathrm{ml}$ of phosphate-buffered saline with $500 \mu \mathrm{g} / \mathrm{mL}$ of the transcription inhibitor rifampicin (PBS + Rif) in one $5 \mathrm{~mL}$ cytometer tube, and incubated during 1 hour in a water bath at $37^{\circ} \mathrm{C}$, so that transcription kept blocked and GFP had time to mature and fold properly. Samples were measured at different time points using the BD FACSCalibur flow cytometer (original default configuration parameters), with and without adding $\mathrm{AHL}_{\mathrm{e}}$ as external 
A

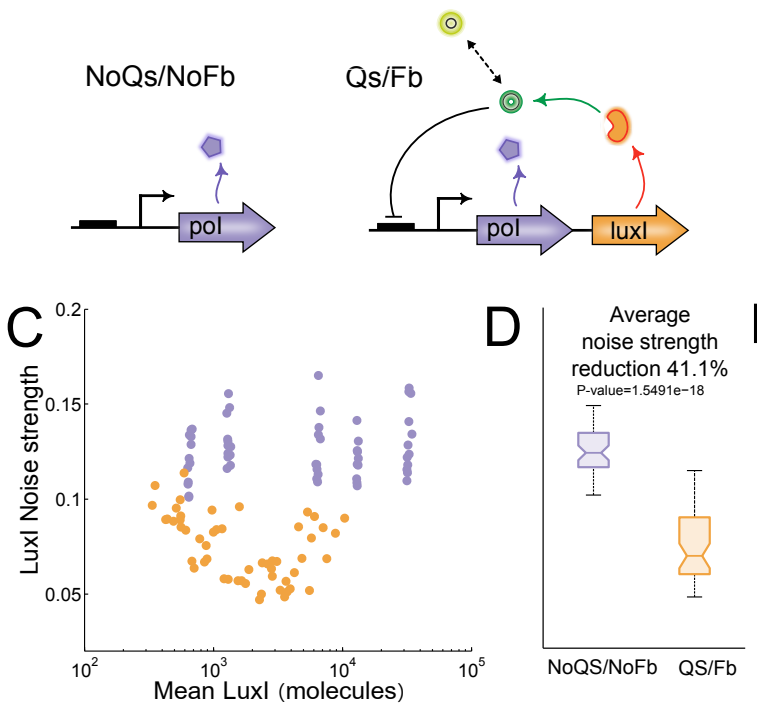

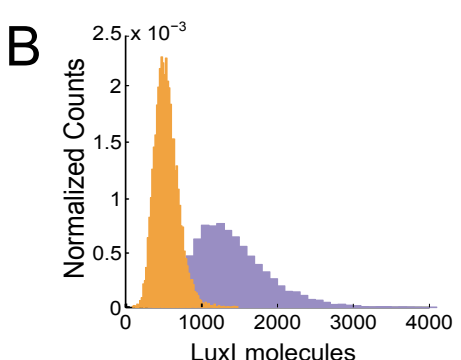

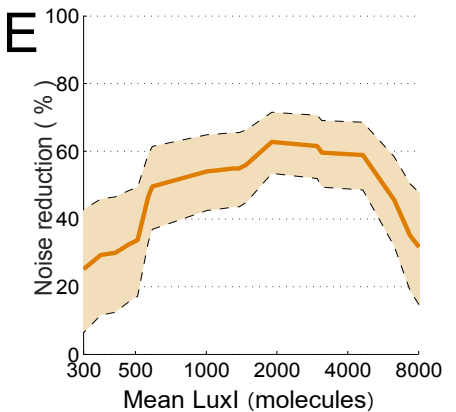

Figure 2. LuxI noise strength under presence/absence of quorum sensing and negative feedback. A. Circuits topologies: NoQS/NoFb (top) and QS/Fb (bottom). B. Representative computational population histograms of LuxI noise strength for $\mathrm{QS} / \mathrm{Fb}$ (orange) presenting a narrower gaussian-like distribution as compared to the Poisson-like one of NoQS/NoFb (purple). C. Sampled combinations of LuxI expression characteristics for fixed LuxR ones show larger values of LuxI noise strength versus mean for NoQS/NoFb (purple dots) than for QS/Fb (orange dots) D. The QS/Fb circuit significantly reduces the average noise strength for the sampled parameters space by $41 \%$, from $\left\langle\eta_{\mathrm{NoQS} / \mathrm{NoFb}}^{2}\right\rangle=0.1263$ down to $\left\langle\eta_{\mathrm{QS} / \mathrm{Fb}}^{2}\right\rangle=0.0744$. E. For varying LuxR parameters the average reduction of noise strength in LuxI ranges from $30 \%$ up to $60 \%$ and shows dependence on the mean expression level.

disturbance (10nM N-3-Oxohexanoyl-L-homoserine lactone, Santa Cruz Biotecnology Catalog Number SC205396).

\section{Results}

\section{Quorum sensing and negative feedback attenuate gene expression noise.}

We first addressed the question whether the proposed QS/Fb circuit effectively reduces noise strength with respect to the circuit NoQS/NoFb. Recall the last one consists of the LuxR expression on the one hand, and the protein of interest (PoI) downstream the $\mathrm{p}_{\text {LuxR }}$ repressible promoter, without the luxI gene coding for LuxI protein, on the other (see Fig 2A). Since no autoinducer $A H L$ is neither produced nor externally introduced, there is no repression, so expression of $\mathrm{PoI}$ is essentially a constitutive one. This corresponds to the Poisson distribution observed in the population histograms in Fig $2 \mathrm{~B}$. Contrarily, the QS/Fb histogram departs from the Poisson distribution to become a narrow Gaussian-like one. This fact, and the reduction in the mean expression value, indicate the strong presence of regulation. In both cases we used the nominal parameters (see Table 1).

Reduction in noise strength was not due to a particular choice of the circuit 
parameters, but a property of the proposed topology. Fig $2 \mathrm{C}$ depicts LuxI noise strength versus mean expression for 60 different combinations of the $\mathrm{p}_{L u x R}$ repressible promoter characteristics (see methods section) for both $\mathrm{Qs} / \mathrm{Fb}$ (orange points) and $\mathrm{NoQs} / \mathrm{NoFb}$ (purple points). The points in the figure correspond to the mean values across the cells population for each parameters combination. The magnitude of the noise strength reduction was larger for medium values of mean protein expression. Noise strength levels were similar for all mean expression values in the case of the NoQs $/ \mathrm{NoFb}$ circuit. Mean expression values in this case depend only on the translation rate $\mathrm{p}_{\mathrm{I}}$ for which five discrete values were used, inducing the five mean values seen in the figure. On the contrary, the Qs/Fb circuit showed lower values of noise strength and more graded values of the mean expression level, for it depends on the combination of all three parameters varied.

More importantly, the noise strength was consistently lower for the Qs/Fb circuit. Taking together all the different combinations of promoter parameters for each circuit, the average noise strength was significantly reduced by $41 \%$ in the presence of quorum sensing and negative feedback, decreasing from $\eta_{\mathrm{NoQS} / \mathrm{NoFb}}^{2}=0.1263$ down to $\eta_{\mathrm{QS} / \mathrm{Fb}}^{2}=0.0744$, as shown in Fig $2 \mathrm{P}$.

For given fixed LuxR expression parameters, the noise strength reduction in LuxI showed a clear dependence on its mean expression level. To elucidate if this dependence was only due to the choice of the $\mathrm{p}_{\text {LuxR }}$ promoter parameters we evaluated, for each mean expression level, the ratio between the noise strength of the $\mathrm{Qs} / \mathrm{Fb}$ circuit for all the range of $\mathrm{p}_{\text {LuxR }}$ promoter parameters, and that of the NoQs/NoFb circuit. With this, we obtained the results plotted in Fig $2 \mathrm{E}$ showing the minimum and maximum values of LuxI noise variance reduction as a function of its mean value. In the range between between 600 and 6000 LuxI molecules it was possible to reduce the noise variance at least in $35 \%$ in the worst case scenario, with a maximum reduction of around $70 \%$ for means between 2000 and 3000 molecules.

\section{Feedback pays-off when extrinsic noise dominates.}

At this point the question arises as to what are the roles of quorum sensing and that of feedback in noise strength reduction, and what are their effect in view of both intrinsic and extrinsic noise.

To answer this question we first contextualized the computational results using available experimental data of noise strength and protein abundance in $E$. coli. We used experimental data taken from [90], and plotted it against our computational results in three scenarios: base control circuit with no quorum sensing nor feedback (NoQS/NoFb, $\left.k_{A}=0\right)$, our circuit with both quorum sensing and feedback $(\mathrm{QS} / \mathrm{Fb})$, and the hypothetical circuit with feedback but without quorum sensing (NoQS/Fb, $D=0$ ). For each scenario we considered different combinations of parameters under the same conditions as in section, with values of the mean protein number in the range $10^{0}-10^{5}$.

Fig 3 A shows the experimental data plotted as black dots. The dashed red and blue lines are the intrinsic and extrinsic noise limits respectively, taken from the same reference. Simulations including both intrinsic and extrinsic noise are plotted as purple dots (NoQS/NoFb), green (NoQS/Fb) and orange ones (QS/Fb) using the same data as in Fig 2C. Our computational results fully agreed with the experimental data and derived limits in 90 . The results corresponding to the base control circuit NoQS/NoFb clearly were over the noise limits.

Unexpectedly, noise strength of both circuits QS/Fb and NoQS/Fb integrating feedback showed very similar behavior. As shown in Fig $3 \mathrm{~B}$ the QS/Fb and NoQS/Fb points lay in the same region. For medium and high mean protein expression values noise strength in $\mathrm{QS} / \mathrm{Fb}$ and $\mathrm{NoQS} / \mathrm{Fb}$ decreased just below the reported extrinsic noise 


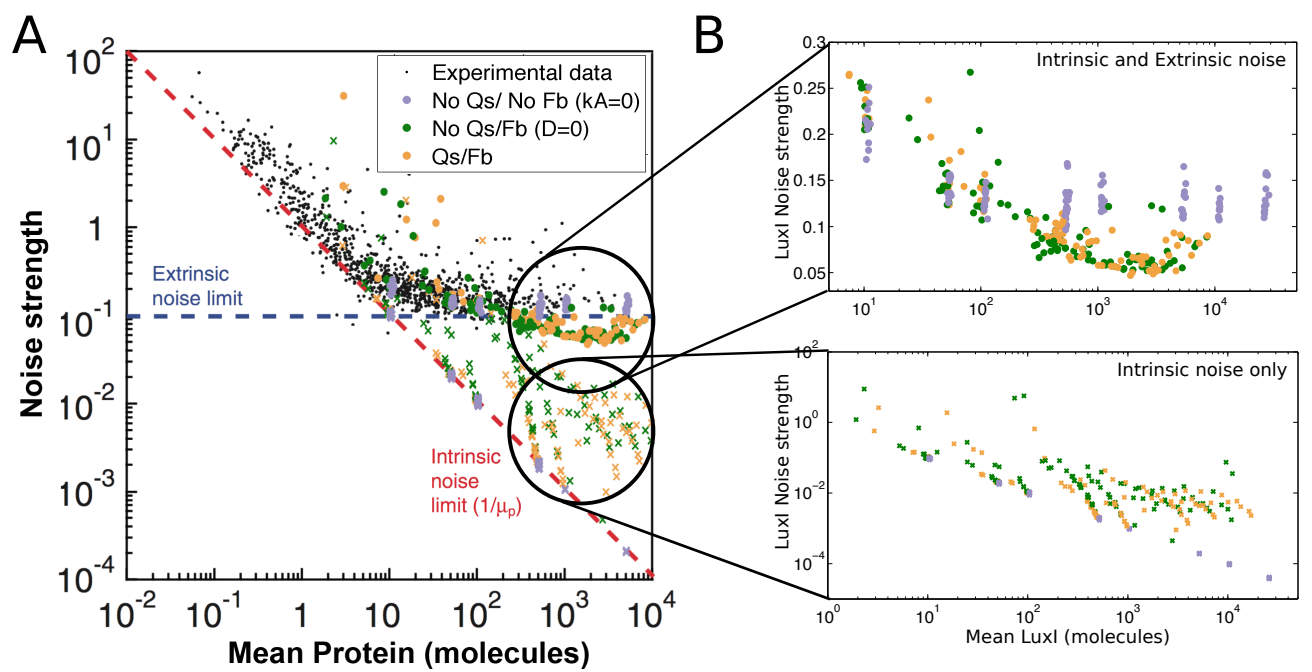

Figure 3. Comparison between experimental data and different scenarios evaluated computationally. A. Experimental data of protein abundance and noise in E. coli taken from [90] is plotted as black dots. The dashed red and blue lines are the intrinsic noise limit and the extrinsic noise limits respectively, taken from the same reference. Simulations of the gene circuits in our study, including both intrinsic and extrinsic noise, are plotted using purple dots (NoQS/NoFb), green (NoQS/Fb) and orange ones $(\mathrm{QS} / \mathrm{Fb})$. Simulations including only intrinsic noise are plotted as crosses: violet (NoQS/NoFb), green (NoQS/Fb) and orange (QS/Fb). B. Zoom of the scenarios considering both intrinsic and extrinsic noise (top) and only intrinsic noise (bottom).

limit, and well below the noise strength for the base NoQS/NoFb circuit. Though high protein expression are of main interest for the intended application of our circuit in an industrial biotechnological context of heterologous protein production, we were interested in the performance of the circuits at low mean protein numbers. Interestingly, the situation in the region was reversed. The open loop circuit NoQS/NoFb showed consistent lower noise strength values than QS/Fb and NoQS/Fb. Therefore, feedback contributed to reducing noise strength for medium-high protein expression where extrinsic noise dominates.

\section{Quorum sensing helps feedback to cope with intrinsic noise.}

The last result was inconclusive about the contribution of quorum sensing to reduce noise strength. To settle this issue we concentrated our analysis in the medium-high protein expression region where feedback contributed to reduce noise strength and extrinsic noise dominates.

We first wanted to elucidate whether QS mainly contributed reducing the intrinsic component of noise. If this was the case, its effect could be masked by the dominant extrinsic noise. To that end we carried out simulations for the same combinations of parameters as before, but suppressing extrinsic noise, and considering the three scenarios NoQS/NoFb, QS/Fb, and NoQS/Fb. The results are shown in Fig 3A, plotted as violet (NoQS/NoFb, $k_{A}=0$ ), green (NoQS/Fb, $D=0$ ) and orange crosses (QS/Fb). Fig $3 \mathrm{~B}$ shows a zoom into the relevant region. Introducing either feedback alone or feedback plus quorum sensing increased noise strength values with respect to the minimal base control circuit representing plain constitutive protein expression. The results for this base NoQS/NoFb circuit were along the experimental intrinsic noise 
limit derived in 90 . These results were consistent with the findings at low mean protein values where intrinsic noise dominates. The circuit NoQS/Fb with feedback and no cell-to-cell communication showed higher values of noise strength, specially for lower values of mean protein number. Finally, reintroducing quorum sensing (QS/Fb) was able to slightly improve noise strength.

To confirm this result we evaluated the difference between the noise strength in LuxI between the circuits $\mathrm{QS} / \mathrm{Fb}$ and $\mathrm{NoQS} / \mathrm{Fb}$ when only intrinsic noise is present as a function of circuit parameters associated to LuxI expression. Fig 4(A) shows the noise strength map difference for different combinations of the dissociation constant $\mathrm{k}_{\mathrm{dlux}} \mathrm{vs}$. the LuxI translation rate $\mathrm{p}_{\mathrm{I}}$ when we consider a tight promoter $\mathrm{P}_{\text {lux }}, \alpha=0.01$ or a leaky one $\alpha=0.1$ in both noise scenarios. The noise strength reduction when QS was added reached a $200 \%$ for low values of $\mathrm{p}_{\mathrm{I}}$. Increasing the dissociation constant improved the reduction, specially for a leaky promoter.

The previous result suggested that the results reported in the literature showing a reduction in noise strength when QS was used were a result of modeling extrinsic noise as an additive signal. This hypothesis was confirmed when besides intrinsic noise we introduced an additive extrinsic noise to our system, with variance independent of the system states. Fig 4(B) shows that in this case there also was a generalized noise strength reduction for most parameter combinations.

Finally, in case we restored extrinsic noise as parametric variability the results showed that adding QS may increase or decrease noise strength (Fig $4(\mathrm{C})$ ) strongly depending on the values of the circuits parameters, and suggesting that getting benefit of QS for medium-large mean expression values requires optimizing the circuit parameters tuning.

\section{Tuning LuxI expression allows minimising noise-strength.}

Dependence of mean expression and noise strength on the Qs/Fb circuit parameters is a key factor to understand for it to be of potential practical usage. To this end we performed thorough in silico experiments to estimate the noise strength and mean expression value of LuxI, as a proxy of the protein of interest, for different sets of the circuit parameters associated to LuxI expression as described in the methods section . We only evaluated two values for the basal expression, corresponding to a tight $\mathrm{P}_{\text {lux }}$ promoter $(\alpha=0.01)$, and a leaky one $(\alpha=0.1)$. As for LuxR, we also considered two values: a strong $\mathrm{RBS}\left(\mathrm{p}_{\mathrm{R}}=10\right)$, and a medium-weak one $\left(\mathrm{p}_{\mathrm{R}}=2\right)$. We kept all other parameters to their nominal values described in table 1. Recall that although we considered variations in the translation rates $\mathrm{p}_{\mathrm{I}}$ and $\mathrm{p}_{\mathrm{R}}$, these are tantamount to considering equivalent variations in the lumped values of the corresponding products of protein burst size, transcription rate and gene copy number.

Fig 5 shows the noise strength map for different combinations of the dissociation constant $\mathrm{k}_{\mathrm{dlux}}$ vs. the LuxI translation rate $\mathrm{p}_{\mathrm{I}}$ when we consider both a tight promoter $\mathrm{P}_{\text {lux }}, \alpha=0.01($ Fig $5 \mathrm{~A})$ and or a leaky one $\alpha=0.1$ (Fig $\left.5 \mathrm{~B}\right)$. The means of LuxI protein number are shown as contour lines.

The mean expression levels of LuxI followed general monotonous trends in all cases. It increased for simultaneous increasing of the dissociation constant and the LuxI translation rate. On the other hand, increasing leakiness of the LuxI promoter did tend to lower mean expression levels of LuxI for low values of the dissociation constant. Finally, using a weaker RBS controlling the translation of LuxR (Fig 5B) produced a steeper increasing of the mean expression level as the dissociation constant and the LuxI translation rate increase.

Noise strength did not show simple patters as a function of the circuit parameters. Larger variations between high and low noise strength values were observed for stronger LuxR RBS (Fig 5A) independent of the leakiness of the promoter $\mathrm{P}_{\text {lux }}$. In this case, the 


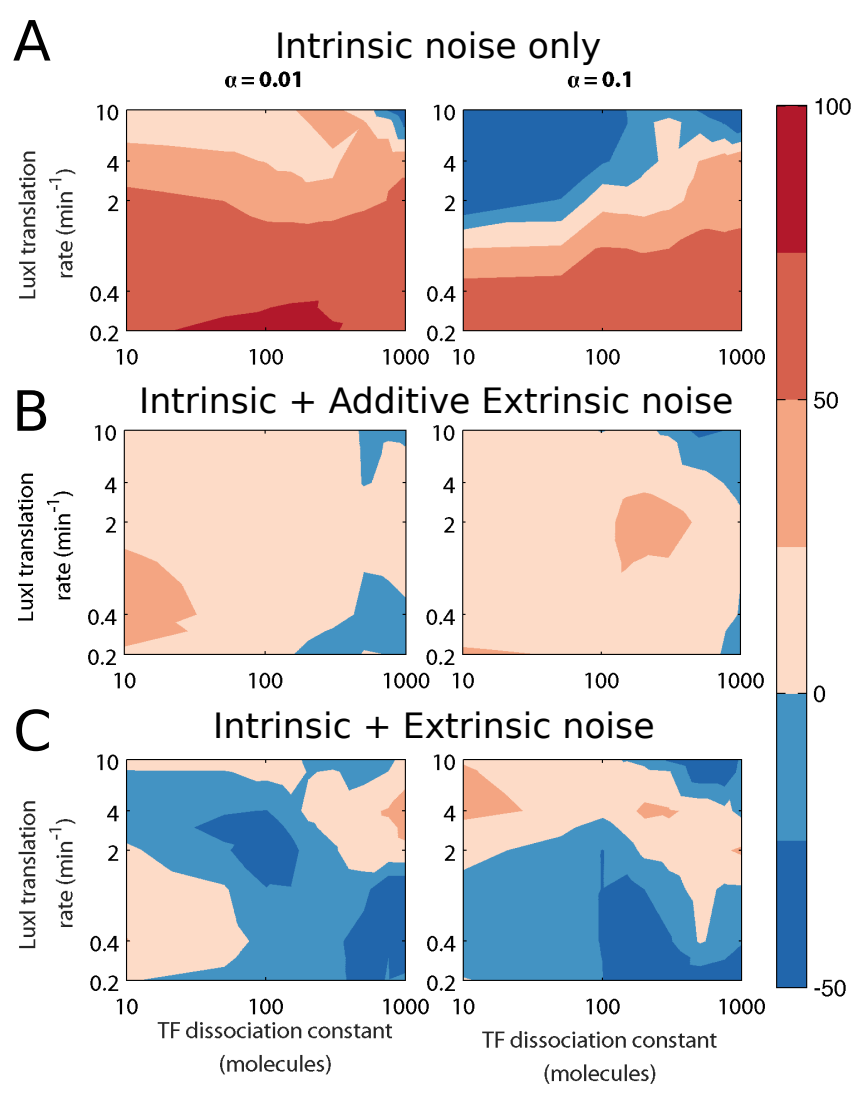

Figure 4. LuxI noise strength reduction as a function of circuit parameters. Color map of the reduction of LuxI noise strength when QS is added to Fb w.r.t. the dissociation constant $\mathrm{k}_{\mathrm{dlux}}$ and the LuxI translation rate $\mathrm{p}_{\mathrm{I}}$. All other parameters were set to their nominal values. Left) Tight promoter $\alpha=0.01$. Right) Leaky promoter $\alpha=0.1$. A) Only intrinsic noise is present. B) Intrinsic noise is present incorporating also additive extrinsic noise. C) Intrinsic and extrinsic noise present.

lowest values of noise strength were achieved for values of the dissociation constant $\mathrm{k}_{\mathrm{dlux}}$ in the range [100 - 500], and values of LuxI translation rate $\mathrm{p}_{\mathrm{I}}$ in the range [2-10]. The mean expression levels in this region were between $2 \cdot 10^{3}$ and $4 \cdot 10^{3}$ proteins, in agreement with the results shown in Fig 2 Decreasing the LuxR RBS strength kept the the values of minimal noise strength essentially in the same region, but with higher values $(\mathrm{Fig} 5 \mathrm{~B})$. The same trend towards higher values of noise strength was observed when the tight promoter $\mathrm{P}_{\text {lux }}$ was changed for a leaky one. This was more evident when a stronger LuxR RBS was used (Fig 5 A).

\section{Fast LuxR turnover reduces LuxI noise strength.}

Next we analyzed in more detail the effect of LuxR expression parameters on LuxI mean expression level and noise strength. In particular, we were interested in the effect of the LuxR translation rate $p_{R}$, as main tuning knob of the LuxI mean expression level, and the one of the degradation rate $d_{R}$, as it has been suggested that fast LuxR turnover can reduce the extrinsic noise. We fixed the LuxI traslation rate to two values $p_{I}=2$ and $p_{I}=2\left[\mathrm{~min}^{-1}\right]$ around its nominal value, and again considered both a tight pLux promoter $(\alpha=0.01)$ and a a leaky one $(\alpha=0.1)$. We kept all other parameters to 

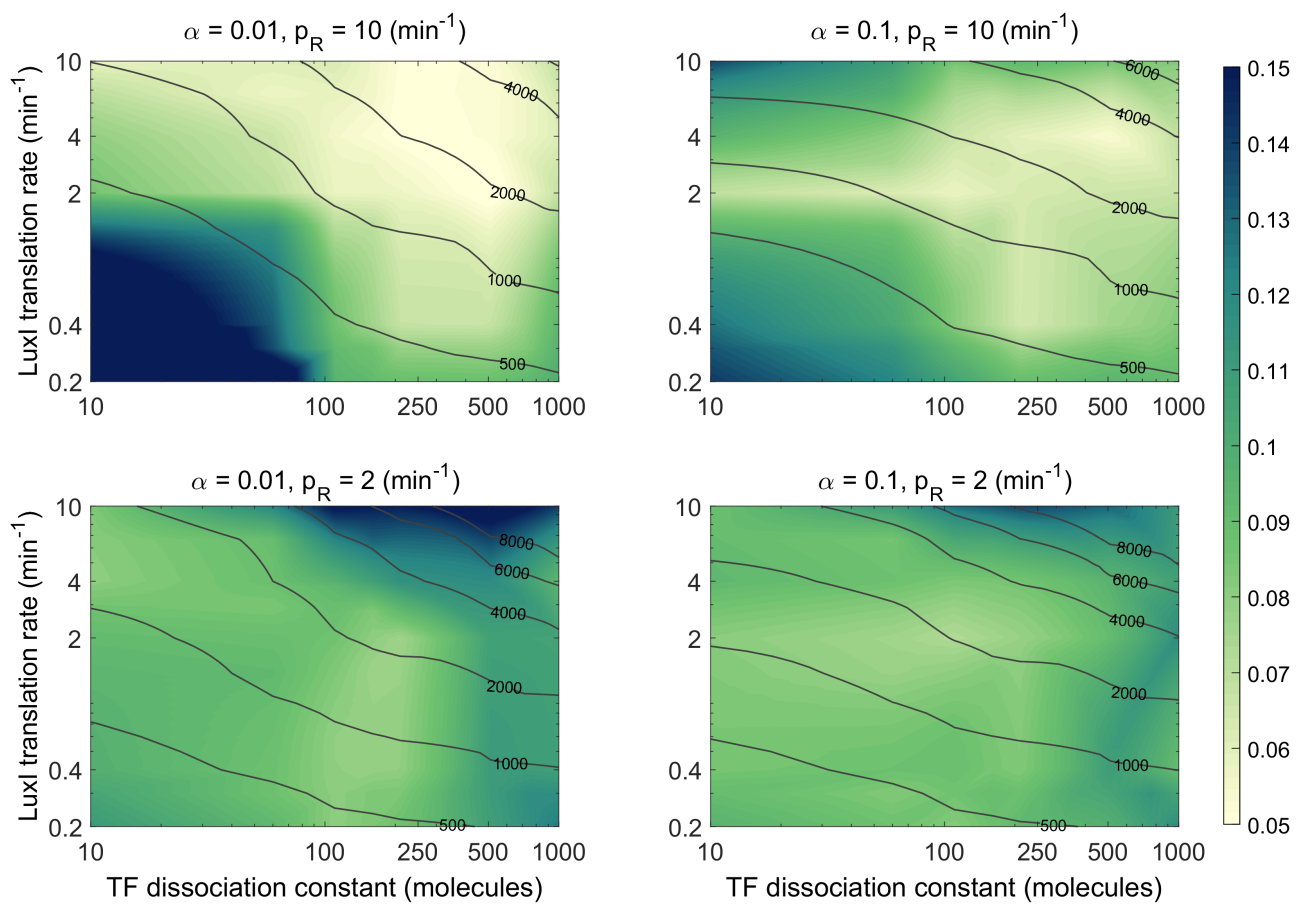

Figure 5. LuxI noise strength and mean as a function of circuit parameters. Color map of LuxI noise strength w.r.t. the dissociation constant $\mathrm{k}_{\mathrm{dlux}}$ and the LuxI translation rate $\mathrm{p}_{\mathrm{I}}$. The level curves correspond to the mean number of LuxI molecules. A) Strong LuxR RBS with $\mathrm{p}_{\mathrm{R}}=10$ [1/min]. B) Medium-weak LuxR RBS with $\mathrm{p}_{\mathrm{R}}=2$ $[1 / \mathrm{min}]$.

their nominal values described in table 1 ,

Fig 6 shows the LuxI noise strength maps and mean expression level curves as a function of values of the LuxR translation rate in the range 0.2 to $101 / \mathrm{min}$, and LuxR degradation rate in the range 0.02 to $0.21 / \mathrm{min}$. The mean expression level did depend little on the LuxR degradation rate, with a slight increase for large ones. LuxR translation rates or, tantamount, LuxR synthesis rates, proved to be a good sensitive tool to tune the desired LuxI mean expression rate, with larger values of the last as the former decreased.

Interestingly, LuxI noise strength decreased as LuxR degradation rate increased, with optimal values in the range 0.07 to $0.21 / \mathrm{min}$ ( $c f$. nominal value in table 1). It did not show a clear dependence on LuxR translation rate, but for an interesting region, for values of LuxR around its nominal value, where it tended to decrease for all values of the degradation rate.

\section{Experimental results confirm computational predictions.}

Experimental implementation of the proposed QS/Fb circuit would not only allow a preliminary experimental validation of its capability to reduce noise strength, but would also further validate the model parameters used throughout this study. Recall the comparison in Fig 3 referred our results to the general landscape of mean protein abundances and noise strengths in $E$ coli. To this end we experimentally implemented both the NoQS/NoFb and QS/Fb circuits as described in section, and compared the experimental results with the ones obtained using the corresponding nominal 

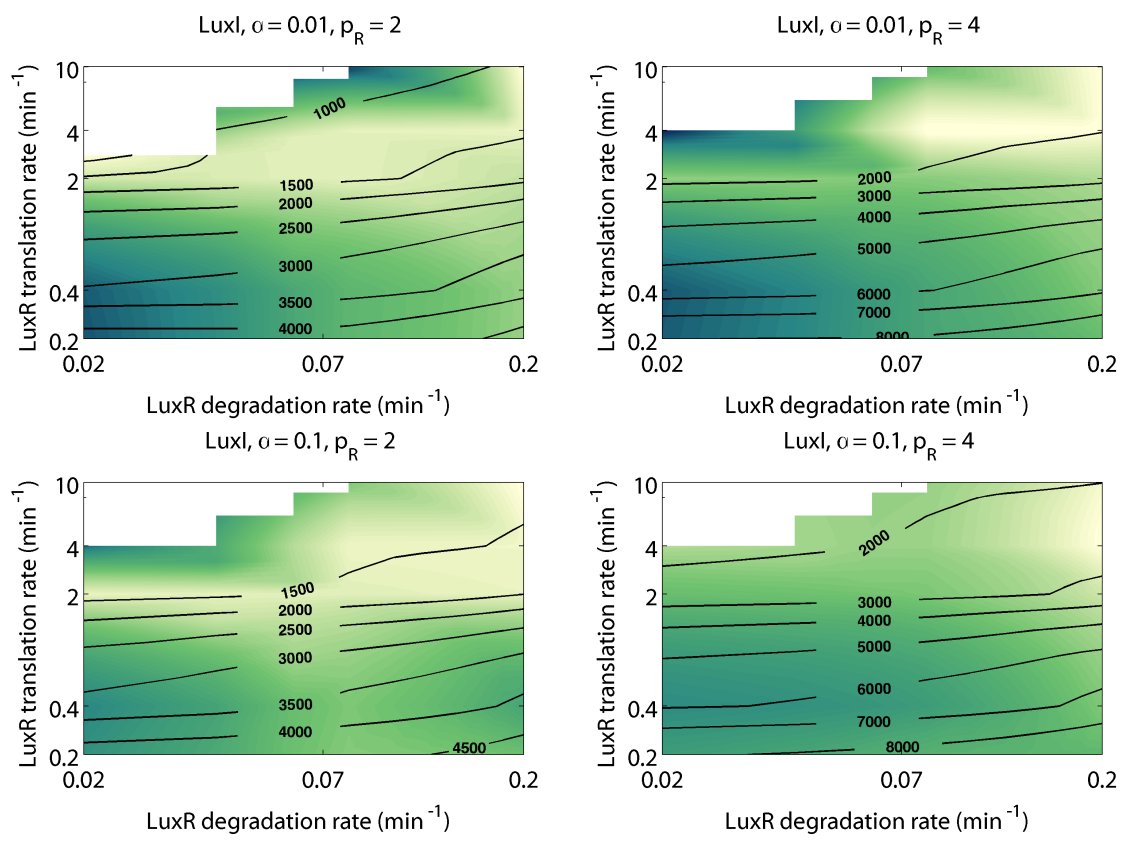

Figure 6. LuxI noise strength versus LuxR parameters. LuxI noise strength maps and mean expression level curves for a tight pLux promoter $(\alpha=0.01$, top) and a a leaky one $\left(\alpha=0.1\right.$, bottom) with LuxI translation rates $p_{I}=2$ (left) and $p_{I}=4$ (right) around its nominal value.
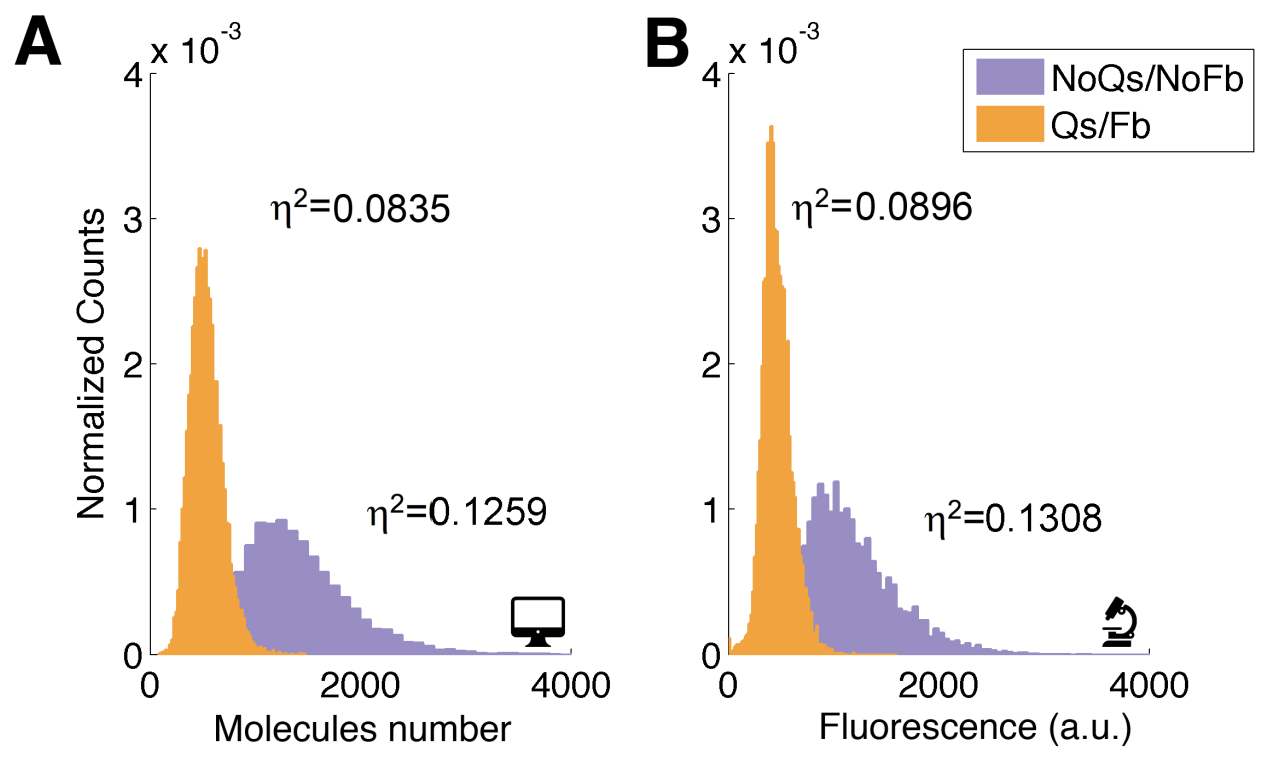

Figure 7. Comparison between experimental and computational results. Population distributions and noise strength for both Qs/Fb and NoQs/NoFb circuits. The plots in A correspond to the computational models simulation, and the ones in $\mathrm{B}$ correspond to experimental flow cytometry results. 
computational models.

The steady state population histograms of LuxI for the circuits Qs/Fb (orange) and NoQs/NoFb (purple) under the same experimental conditions are depicted in Fig 7 . The computational predictions for the nominal models are in the panel A, while the B panel shows flow cytometry experimental results. Both results were qualitatively comparable without any tuning, fitting or change in the model parameters. We only required a common scaling factor to convert from relative units of fluorescence to number of molecules. The experimental results showed LuxI noise strength is reduced by $31.5 \%\left(\eta_{Q s / F b}^{2}=0.0896, \eta_{N o Q s / N o F b}^{2}=0.1308\right)$. On the other hand, the computational simulations predicted a $33.6 \%$ reduction $\left(\eta_{Q s / F b}^{2}=0.0835, \eta_{N o Q s / N o F b}^{2}=0.1259\right)$.

As we expected, the mean expression level in the NoQs/NoFb circuit, $\mu_{\text {NoQs } / \text { NoFb }}=1166.46$ (a.u.) (simulation $\mu_{\text {NoQs } / \text { NoFb }}=1395.65$ (molecules)), was larger than the one in the Qs $/ \mathrm{Fb}$ circuit, $\mu_{Q s / F b}=462.63$ (a.u.) (simulation $\mu_{Q s / F b}=533.67$ (molecules)). Indeed, the circuit Qs/Fb incorporates a feedback loop that changes the location of equilibrium point with respect to the open loop circuit. To test whether the reduction in noise strength is an effect associated with the reduction in the mean expression level, we compared both circuits applying an induction of $3 \mathrm{nM}$ AHL to the the open loop NoQs/NoFb circuit. With this induction, GFP fluorescence mean values of both circuits was comparable.

\section{Discussion}

Our results show that gene synthetic circuits benefiting from the interplay between feedback and cell-to-cell communication allow control of the mean expression level and noise strength of a protein of interest. A few circuit parameters easy to tune in the wet-lab can be used to achieve noise strength reductions up to a $60 \%$ with respect to constitutive expression of the protein of interest.

Mean expression level and noise strength are not independent goals. At low mean values intrinsic noise dominates and sets the minimum noise strength attainable. At high mean values extrinsic noise dominates. Thus, there is a trade-off between expression level and noise strength, as revealed both by system-wide experimental data and theoretical analysis reported in the literature. Our computational results fitted well in this scenario, and suggest that tuning synthetic gene circuits to minimize noise while achieving a desired expression level will require a multi-objective optimization approach.

For high mean expression values we observed a clear benefit of having feedback as compared to constitutive expression. Yet, adding quorum sensing on top of feedback did not decrease noise strength unless the circuit parameters are tuned. That is, the benefit from adding cell-to-cell communication is not structural, but depended on proper choice of the circuit parameters. This result is somewhat counter-intuitive and does not fully agree with previous works reporting a reduction of extrinsic noise in quorum sensing-based gene circuits, e.g. 20, that reported a structural benefit. This may be explained by the different approaches to model extrinsic noise. While we modeled it as parametric variability, most often extrinsic noise has been modeled as an additive stochastic signal essentially analogous to the intrinsic noise term. Thus, if we considered a scenario with intrinsic noise and no extrinsic one while keeping medium-high expression means, our results also showed an important reduction of noise strength when quorum sensing was added to feedback. Though the amount of reduction depended on the circuit parameters, we observed noise reduction for almost any combination of them. Moreover, if we considered additive extrinsic noise, we got qualitatively similar results to the ones when only intrinsic noise was present.

In the hypothetical scenario with no extrinsic noise we also found that adding either feedback or feedback and quorum sensing increased the noise strength with respect to 
the open loop constitutive gene expression circuit. This result might be explained by the increased complexity introduced by these circuits [91]. Yet, circuit complexity is not the only factor contributing. On the one hand, the circuit with quorum sensing and feedback achieved lower average noise strength values than the less complex only-feedback one in this scenario. On the other, when extrinsic noise was present constitutive expression was clearly noisier than any of the more complex Qs/Fb and NoQs/Fb circuits for high protein mean expression values, though not for low ones where intrinsic noise dominates. Thus, the circuit complexity contribution to noise depends not only on its size, but in the interplay between size and noise structure. Thus, in the medium-high range of mean protein expression, of interest for industrial biotechnology, tuning circuit parameters in the circuit with both quorum sensing and feedback clearly allows coping with both intrinsic noise and extrinsic one, however its structure.

The experimental results, though preliminary, showed a high concordance the computational ones and confirmed the capability of the proposed circuit to reduce noise strength.

\section{Acknowledgments}

Research in this area is partially supported by Spanish government (CICYT DPI2014-55276-C5-1) and European Union (FEDER). Y. Boada thanks grant FPI/2013-3242 of UPV.

\section{References}

1. Raser JM, O'Shea EK. Noise in gene expression: origins, consequences, and control. Science. 2005;309(5743):2010-2013.

2. Kærn M, Elston TC, Blake WJ, Collins JJ. Stochasticity in gene expression: from theories to phenotypes. Nat Rev Genet. 2005;6(6):451-64.

3. Fraser HB, Hirsh AE, Giaever G, Kumm J, Eisen MB. Noise minimization in eukaryotic gene expression. PLoS Biol. 2004 6;2(6):e137.

4. Eldar A, Elowitz MB. Functional roles for noise in genetic circuits. Nature. 2010;467(7312):167-173.

5. Raj A, van Oudenaarden A. Nature, nurture, or chance: stochastic gene expression and its consequences. Cell. 2008;135(2):216-226.

6. Labhsetwar P, Cole JA, Roberts E, Price ND, Luthey-Schulten ZA. Heterogeneity in protein expression induces metabolic variability in a modeled Escherichia coli population. Proc Natl Acad Sci U S A. 2013 8;.

7. Acar M, Mettetal JT, van Oudenaarden A. Stochastic switching as a survival strategy in fluctuating environments. Nature genetics. 2008;40(4):471-475.

8. Elowitz MB, Levine AJ, Siggia ED, Swain PS. Stochastic gene expression in a single cell. Science. 2002 8;297(5584):1183-6.

9. Ozbudak EM, Thattai M, Kurtser I, Grossman AD, van Oudenaarden A. Regulation of noise in the expression of a single gene. Nat Genet. 2002 5;31(1):69-73.

10. Cai L, Friedman N, Xie XS. Stochastic protein expression in individual cells at the single molecule level. Nature. 2006 3;440(7082):358-62. 
11. Chalancon G, Ravarani CNJ, Balaji S, Martinez-Arias A, Aravind L, Jothi R, et al. Interplay between gene expression noise and regulatory network architecture. Trends Genet. 2012 5;28(5):221-32.

12. Murphy KF, Adams RM, Wang X, Balázsi G, Collins JJ. Tuning and controlling gene expression noise in synthetic gene networks. Nucleic Acids Res. 2010 $5 ; 38(8): 2712-26$.

13. Jones DL, Brewster RC, Phillips R. Promoter architecture dictates cell-to-cell variability in gene expression. Science. 2014 12;346(6216):1533-6.

14. Swain PS, Elowitz MB, Siggia ED. Intrinsic and extrinsic contributions to stochasticity in gene expression. Proceedings of the National Academy of Sciences. 2002;99(20):12795-12800.

15. Singh A, Hespanha JP. Optimal feedback strength for noise suppression in autoregulatory gene networks. Biophys J. 2009;96(10):4013-23.

16. Toni T, Tidor B. Combined model of intrinsic and extrinsic variability for computational network design with application to synthetic biology. PLoS Comput Biol. 2013;9(3):e1002960.

17. Dar RD, Razooky BS, Weinberger LS, Cox CD, Simpson ML. The Low Noise Limit in Gene Expression. PLoS One. 2015;10(10):e0140969.

18. Rao CV, Wolf DM, Arkin AP. Control, exploitation and tolerance of intracellular noise. Nature. 2002;420(6912):231-237.

19. Bruggeman FJ, Blüthgen N, Westerhoff HV. Noise management by molecular networks. PLoS Comput Biol. 2009 9;5(9):e1000506.

20. Tanouchi Y, Tu D, Kim J, You L. Noise reduction by diffusional dissipation in a minimal quorum sensing motif. PLoS Comput Biol. 2008;4(8):e1000167.

21. Tabbaa OP, Nudelman G, Sealfon SC, Hayot F, Jayaprakash C. Noise propagation through extracellular signaling leads to fluctuations in gene expression. BMC Syst Biol. 2013;7:94.

22. Weber M, Buceta J. Noise regulation by quorum sensing in low mRNA copy number systems. BMC Syst Biol. 2011;5:11.

23. Venturi V. Regulation of quorum sensing in Pseudomonas. FEMS microbiology reviews. 2006;30(2):274-291.

24. Miyashiro T, Ruby EG. Shedding light on bioluminescence regulation in Vibrio fischeri. Molecular microbiology. 2012;84(5):795-806.

25. Kaplan HB, Greenberg EP. Diffusion of autoinducer is involved in regulation of the Vibrio fischeri luminescence system. Journal of bacteriology. 1985;163(3):1210-1214.

26. Fuqua C, Parsek MR, Greenberg EP. Regulation of gene expression by cell-to-cell communication: acyl-homoserine lactone quorum sensing. Annual review of genetics. 2001;35(1):439-468.

27. Waters CM, Bassler BL. Quorum sensing: cell-to-cell communication in bacteria. Annu Rev Cell Dev Biol. 2005;21:319-346. 
28. Tabareau N, Slotine JJJ, Pham QCC. How synchronization protects from noise. PLoS Comput Biol. 2010;6(1):e1000637.

29. Nelson EM, Kurz V, Perry N, Kyrouac D, Timp G. Biological Noise Abatement: Coordinating the Responses of Autonomous Bacteria in a Synthetic Biofilm to a Fluctuating Environment Using a Stochastic Bistable Switch. ACS synthetic biology. 2013;.

30. Weber M, Buceta J. Dynamics of the quorum sensing switch: stochastic and non-stationary effects. BMC Syst Biol. 2013;7:6.

31. Geiler-Samerotte KA, Bauer CR, Li S, Ziv N, Gresham D, Siegal ML. The details in the distributions: why and how to study phenotypic variability. Curr Opin Biotechnol. 2013 8;24(4):752-9.

32. Müller S, Harms H, Bley T. Origin and analysis of microbial population heterogeneity in bioprocesses. Curr Opin Biotechnol. 2010 2;21(1):100-13.

33. Fernandes RL, Nierychlo M, Lundin L, Pedersen AE, Puentes Tellez PE, Dutta A, et al. Experimental methods and modeling techniques for description of cell population heterogeneity. Biotechnol Adv. 2011;29(6):575-99.

34. Carlquist M, Fernandes RL, Helmark S, Heins ALL, Lundin L, Sorensen SJ, et al. Physiological heterogeneities in microbial populations and implications for physical stress tolerance. Microb Cell Fact. 2012;11:94.

35. Purnick PEM, Weiss R. The second wave of synthetic biology: from modules to systems. Nat Rev Mol Cell Biol. 2009 6;10(6):410-22.

36. Way JC, Collins JJ, Keasling JD, Silver PA. Integrating biological redesign: where synthetic biology came from and where it needs to go. Cell.

2014;157(1):151-61.

37. Dublanche Y, Michalodimitrakis K, Kümmerer N, Foglierini M, Serrano L. Noise in transcription negative feedback loops: simulation and experimental analysis. Mol Syst Biol. 2006;2:41.

38. Vignoni A, Oyarzún DA, Picó J, Stan GB. Control of protein concentrations in heterogeneous cell populations. In: 2013 European Control Conference (ECC); 2013. p. 3633-3639.

39. Zargar A, Quan DN, Bentley WE. Enhancing Intercellular Coordination: Rewiring Quorum Sensing Networks for Increased Protein Expression through Autonomous Induction. ACS Synth Biol. 2016;5(9):923-8.

40. Zechner C, Seelig G, Rullan M, Khammash M. Molecular circuits for dynamic noise filtering. Proceedings of the National Academy of Sciences. 2016 4;p. 201517109.

41. Heinrich R, Schuster S. The Regulation of Cellular Systems. Chapman and Hall, New York; 1996.

42. Alon U. An Introduction to Systems Biology. Desing Principles of Biological Circuits. Champan and Hall/CRC, Edition; 2007.

43. Chellaboina V, Bhat S, Haddad W, Bernstein D. Modeling and analysis of mass-action kinetics. IEEE Control Systems Magazine. 2009 8;29(4):60-78. 
44. Wilkinson DJ. Stochastic modelling for quantitative description of heterogeneous biological systems. Nat Rev Genet. 2009 2;10(2):122-33.

45. Gillespie DT. A rigorous derivation of the chemical master equation. Physica A: Statistical Mechanics and its Applications. 1992;188(1):404-425.

46. Jahnke T, Huisinga W. Solving the chemical master equation for monomolecular reaction systems analytically. Journal of mathematical biology. 2007;54(1):1-26.

47. Grima R, Schmidt D, Newman T. Steady-state fluctuations of a genetic feedback loop: An exact solution. The Journal of chemical physics. 2012;137(3):035104.

48. Munsky B, Khammash M. The finite state projection algorithm for the solution of the chemical master equation. The Journal of chemical physics. 2006;124(4):044104.

49. Kazeev V, Khammash M, Nip M, Schwab C. Direct solution of the chemical master equation using quantized tensor trains. PLoS computational biology. 2014;10(3):e1003359.

50. Chevalier MW, El-Samad H. A rigorous framework for multiscale simulation of stochastic cellular networks. The Journal of chemical physics.

2009;131(5):054102-054102.

51. Gillespie DT. Stochastic simulation of chemical kinetics. Annu Rev Phys Chem. 2007;58:35-55.

52. Scott M, Ingalls B, Kaern M. Estimations of intrinsic and extrinsic noise in models of nonlinear genetic networks. Chaos. 2006 6;16(2):026107.

53. Picó J, Vignoni A, Picó-Marco E, Boada Y. Modelling biochemical systems: from Mass Action Kinetics to Linear Noise Approximation. Revista Iberoamericana de Automática e Informática Industrial RIAI. 2015 7;12(3):241-252.

54. Gillespie DT. The chemical Langevin equation. The journal of Chemical Physics. 2000;113:297-306.

55. Higham DJ. Modeling and Simulating Chemical Reactions. SIAM Review. 2008;50(2):347-368.

56. Joo J, Plimpton SJ, Faulon JLL. Statistical ensemble analysis for simulating extrinsic noise-driven response in NF- $\mathrm{CB}$ signaling networks. BMC Syst Biol. 2013;7:45.

57. Kim KH, Sauro HM. Adjusting phenotypes by noise control. PLoS Comput Biol. 2012;8(1):e1002344.

58. Russo G, Slotine JJE. Global convergence of quorum-sensing networks. Phys Rev E Stat Nonlin Soft Matter Phys. 2010 10;82(4 Pt 1):041919.

59. Schaefer AL, Val DL, Hanzelka BL, Cronan JE, Greenberg EP. Generation of cell-to-cell signals in quorum sensing: acyl homoserine lactone synthase activity of a purified Vibrio fischeri LuxI protein. Proceedings of the National Academy of Sciences. 1996;93(18):9505-9509.

60. Egland KA, Greenberg EP. Conversion of the Vibrio fischeri Transcriptional Activator, LuxR, to a Repressor. Journal of Bacteriology. 2000;182(3):805-811. 
61. Shih YPP, Wu HCC, Hu SMM, Wang TFF, Wang AHJ. Self-cleavage of fusion protein in vivo using TEV protease to yield native protein. Protein Sci. 2005 4;14(4):936-41.

62. Chen X, Pham E, Truong K. TEV protease-facilitated stoichiometric delivery of multiple genes using a single expression vector. Protein Sci. 2010 $12 ; 19(12): 2379-88$.

63. Zhang B, Rapolu M, Liang Z, Han Z, Williams PG, Su WW. A dual-intein autoprocessing domain that directs synchronized protein co-expression in both prokaryotes and eukaryotes. Sci Rep. 2015 2;5:8541.

64. Zagaris A, Kaper HGG, Kaper TJJ. Analysis of the Computational Singular Perturbation Reduction Method for Chemical Kinetics. Journal of Nonlinear Science. 2004;14(1):59-91.

65. Mélykúti B, Hespanha JaP, Khammash M. Equilibrium distributions of simple biochemical reaction systems for time-scale separation in stochastic reaction networks. J R Soc Interface. 2014 8;11(97):20140054.

66. Hancock EJ, Stan GBB, Arpino JAJ, Papachristodoulou A. Simplified mechanistic models of gene regulation for analysis and design. J R Soc Interface. 2015 7;12(108):20150312.

67. Arpino JAJ, Hancock EJ, Anderson J, Barahona M, Stan GBVB, Papachristodoulou A, et al. Tuning the dials of Synthetic Biology. Microbiology. 2013 7;159(Pt 7):1236-53.

68. Boada Y, Vignoni A, Navarro JL, Picó J. Improvement of a CLE Stochastic Simulation of gene synthetic network with quorum sensing and feedback in a cell population. Proceedings 14th annual European Control Conference. 2015;.

69. Milo R, Phillips R, Orme N. Cell Biology by the Numbers. Garland Science; 2016.

70. Urbanowski ML, Lostroh CP, Greenberg EP. Reversible Acyl-Homoserine Lactone Binding to Purified Vibrio fischeri LuxR Protein. Journal of Bacteriology. 2004 1;186(3):631-637.

71. Harman JG. Allosteric regulation of the cAMP receptor protein. Biochimica et Biophysica Acta (BBA)-Protein Structure and Molecular Enzymology. 2001;1547(1):1-17.

72. Buchler NE, Gerland U, Hwa T. Nonlinear protein degradation and the function of genetic circuits. Proceedings of the National Academy of Sciences of the United States of America. 2005;102(27):9559-9564.

73. Goryachev AB, Toh DJ, Lee T. Systems analysis of a quorum sensing network: design constraints imposed by the functional requirements, network topology and kinetic constants. Biosystems. 2006;83(2-3):178-87.

74. Boada Y, Reynoso-Meza G, Picó J, Vignoni A. Multi-objective optimization framework to obtain model-based guidelines for tuning biological synthetic devices: an adaptive network case. BMC Syst Biol. 2016;10(1):27.

75. Kaufmann GF, Sartorio R, Lee SH, Rogers CJ, Meijler MM, Moss JA, et al. Revisiting quorum sensing: discovery of additional chemical and biological functions for 3-oxo-N-acylhomoserine lactones. Proceedings of the National Academy of Sciences of the United States of America. 2005;102(2):309-314. 
76. Roberts C, Anderson KL, Murphy E, Projan SJ, Mounts W, Hurlburt B, et al. Characterizing the effect of the Staphylococcus aureus virulence factor regulator, SarA, on log-phase mRNA half-lives. Journal of bacteriology. $2006 ; 188(7): 2593-2603$.

77. Santillán M, Mackey MC. Dynamic regulation of the tryptophan operon: A modeling study and comparison with experimental data. Proceedings of the National Academy of Sciences. 2001;98(4):1364-1369.

78. Weiss TF. Cellular biophysics. vol. 1. MIT press Cambridge, Mass:; 1996.

79. Nilsson P, Olofsson A, Fagerlind M, Fagerström T, Rice S, Kjelleberg S, et al. Kinetics of the AHL regulatory system in a model biofilm system: how many bacteria constitute a quorum? Journal of molecular biology. 2001;309(3):631-640.

80. Alberts B, Bray D, Hopkin K, Johnson AD, Johnson A, Roberts K, et al. Essential Cell Biology. 3rd ed. Garland Science; 2009.

81. Zucca S, Pasotti L, Politi N, Cusella MG, Magni P. A standard vector for the chromosomal integration and characterization of BioBrick ${ }^{\mathrm{TM}}$ parts in Escherichia coli. Journal of Biological Engineering. 2013;.

82. Basu S, Gerchman Y, Collins CH, Arnold FH, Weiss R. A synthetic multicellular system for programmed pattern formation. Nature. 2005 4;434(7037):1130-4.

83. Woods ML, Leon M, Perez-Carrasco R, Barnes CP. A statistical approach reveals designs for the most robust stochastic gene oscillators. ACS Synth Biol. 2016 2;.

84. Paulsson J. Summing up the noise in gene networks. Nature. 2004 $1 ; 427(6973): 415-8$.

85. Sbalzarini IF, Walther JH, Bergdorf M, Hieber SE, Kotsalis EM, Koumoutsakos P. PPM - A Highly Efficient Parallel Particle-Mesh Library for the Simulation of Continuum Systems. Journal of Computational Physics. 2006;215(2):566-588.

86. Salis HM, Mirsky EA, Voigt CA. Automated design of synthetic ribosome binding sites to control protein expression. Nature biotechnology. 2009;27(10):946-950.

87. Egbert RG, Klavins E. Fine-tuning gene networks using simple sequence repeats. Proceedings of the National Academy of Sciences of the United States of America. $2012 ;$

88. Schmidl SR, Sheth RU, Wu A, Tabor JJ. Refactoring and optimization of light-switchable Escherichia coli two-component systems. ACS synthetic biology. 2014;3(11):820-831.

89. Brewster RC, Jones DL, Phillips R. Tuning promoter strength through RNA polymerase binding site design in Escherichia coli. PLoS Comput Biol. 2012;8(12):e1002811.

90. Taniguchi Y, Choi PJ, Li GWW, Chen H, Babu M, Hearn J, et al. Quantifying E. coli proteome and transcriptome with single-molecule sensitivity in single cells. Science. 2010;329(5991):533-8.

91. Potvin-Trottier L, Lord ND, Vinnicombe G, Paulsson J. Synchronous long-term oscillations in a synthetic gene circuit. Nature. 2016;538(7626):514-517. 